\title{
CLINICAL GOVERNANCE: WILL IT HAVE AN IMPACT ON DOCTORS LOCALLY?
}

\author{
AR Luksza, Medical Director; IR Cumming, Chief Executive \\ Morecambe Bay Hospitals NHS Trust
}

\section{INTRODUCTION}

The term clinical governance was catapulted to prominence in the NHS White Paper of 1997. The term has since entered into common usage and now readily trips off the tongues of doctors and managers alike. But what exactly does it mean and how will it work? Surprisingly, there is little published guidance. In essence, for the first time trust chief executives become responsible for the quality of clinical care provided by their organisation, as well as all other aspects of nonclinical management within it. This new responsibility has surprised clinicians as many NHS chief executives are not from a clinical background. The concept of a chief executive taking ultimate responsibility for the quality of service provided by his/her organisation is not, however, unusual - in almost every other service or business within the UK apart from healthcare this is usual practice. The responsibility of the chief executive is not about judging the clinical performance of clinicians but ensuring the clinical governance agenda is delivered. So what is the agenda? We believe it can be summarised as follows:

- systems to monitor the quality of clinical practice should be in place and functioning properly

- clinical practice should be reviewed regularly, and improved

- doctors and other healthcare professionals should meet standards set out by national professional regulatory bodies.

This paper details our thoughts on implementing this agenda locally and highlights what we believe are local priorities.

\section{WHY BOTHER?}

At a moral level, it seems entirely appropriate that the NHS should strive to provide the best possible care within the resources available. As we are all potential NHS users, we are sure that everyone agrees about the need to feel confident that the care which we and our families receive should be clinically effective and provided at an appropriate standard. Some doctors believe that existing professional self-regulation mechanisms are perfectly adequate. However, the publicity surrounding the shortcomings of the Bristol cardiac surgery and the Kent cytology services has focused the minds of the government and the public on this topic and reinforced the view that greater control is necessary. Another important consideration is the burgeoning cost of medical litigation. During the 1980's the frequency of NHS medical negligence claims rose five-fold while the cost of settlement for each claim went up by 250 per cent. In 1975, the total cost of claims met by the NHS was $£ 1$ million. By 1996 the figure had escalated to $£ 200$ million. Locally, if the Morecambe Bay
Hospitals NHS Trust was to be found fully liable in all our existing outstanding litigation claims (171 in number), the total cost to the NHS would be in excess of $£ 9$ million.

\section{SO WHAT IS CLINICAL GOVERNANCE?}

The essential principle of clinical governance is to assure and improve clinical standards at a local level throughout the NHS. By making trusts directly accountable for quality of care, the government is requiring a fundamental change in attitudes and culture within the NHS. To help achieve this change, several initiatives at national, regional, trust and individual levels are being introduced.

\section{(i) National level}

At a national level, the government has established the National Institute of Clinical Effectiveness (NICE) whose role will be to evaluate the clinical effectiveness of up to 50 treatments or interventions each year. It will co-opt medical experts to collate evidence on particular topics and produce protocols based on perceived best practice. These protocols will form the basis for new national service frameworks which will be disseminated to trusts, who will have the responsibility for local implementation and subsequent monitoring. To ensure local adherence to this national initiative, a second body, the Commission for Health Improvement (CHImP), has been established and will be empowered to visit local trusts to monitor compliance.

In addition to these new organisations there are several established bodies which are influential in dictating standards of practice. These include the Royal Colleges, GMC and the UKCC. It is essential that the energies of all these professional bodies should be harnessed and coordinated so that the trusts receive clear messages about attainable objectives.

(ii) Regional and health authority level

Historically, health authorities have been the major purchasers of care from acute trusts. Contracts have tended to specify quantity rather than quality of care provided. The first seeds of change came with the introduction of GP fundholders who, for the first time, started to write quality conditions into healthcare contracts. Whilst most of these conditions related to nonclinical quality, eg waiting times, there were certainly many examples where fundholders moved their contracts to alternative providers to achieve what was perceived as a better deal for their patients. In the future, agreements which the health authority or primary care groups/trusts enter into with acute trusts will not only dictate quantity but, more and more, start to specify the quality of the care to be provided. 
(iii) Trust level

At trust level we need to build on systems already in place to achieve quality improvement. Such systems incorporate clinical audit, risk management, the introduction of evidence-based clinical practice, continuing education for all staff, audit of consumer feedback, health needs assessment, development of more and better guidelines and protocols, effective management of poor clinical performance, accreditation and continuing professional development. It is the duty of the trust to ensure that mechanisms are in place to ensure that all the above systems are functioning effectively. Table 1 illustrates the various groups established by this trust as the first stage of our approach to implementing clinical governance. The following specifies a few of these components in greater detail:-

Clinical audit This has been a compulsory component of doctors' duties for several years. Unfortunately its implementation has been patchy. Audits have generally been driven by particular interest groups and the audit loops were rarely closed. In the future it will be necessary to have many more directed audits dictated either by the NICE or the trust board. Systems will have to be introduced to ensure that particular audits are revisited to ensure improvements in clinical practice.

In addition, trusts will be expected to participate in existing national audits such as CEPOD, maternal deaths, suicides and intensive care.

Clinical risk management Many trusts already operate clinical and non-clinical risk management groups. To date, the main function of many of these groups has been merely to collect data from critical incident reports to submit to CNST (the trust's insurers). Within our own trust several directorates already operate a system of critical incident reporting. This practice will need to be extended to all directorates, not only to comply with CNST standards but more importantly so that the organisation may learn from such "near misses". It is important that all reported incidents are collated and that lessons learnt become widely disseminated throughout the trust. Additionally it must be emphasized that critical incident reporting is seen as an educational activity and not as ammunition for potential disciplinary action. Everyone, doctors included, makes mistakes and rather than hiding the evidence we must use it to educate others. Clearly, such a change in culture will be evolutionary but it is essential if clinical governance is to be successful.

As well as critical incident reporting, the clinical risk group would be able to direct systematic risk assessment in areas where mistakes are either frequent or costly in terms of human suffering or litigation. If we take the example of the tragedy of a child born with brain damage, then it would be valuable to look at the various strands of care involved in seeing a pregnant woman from the late stages of pregnancy through to the birth of her baby and to examine all the potential pitfalls to see what changes in practice can be introduced to minimise future risk. Such exercises, whilst valuable, are time-consuming and costly and the question of resources will be considered later in this paper.

Continuing Professional Development (CPD) The lynchpin of clinical governance is continuous learning. Although consultants already partake in CPD, it is too often focused on their specialist interests rather than the wider needs of the trust or their general patient population. Take the medical director as an example, who is employed as a general physician with a special interest in respiratory medicine. His job plan gives a workload equally distributed between general medicine and respiratory medicine. A review of his study leave programme for the last three years suggests that $80 \%$ has been centred on updating his knowledge of respiratory medicine with only a minority covering the much wider field of general medicine.

The trust may now need to move towards a more formal system of appraisal for consultants, which not only concentrates on job plans but also extends to matters of educational needs and professional performance. Although there is already an obligation to review job plans annually, the task has only been given lip service and has rarely resulted in any change. In future, appraisal may incorporate a review of doctors' service

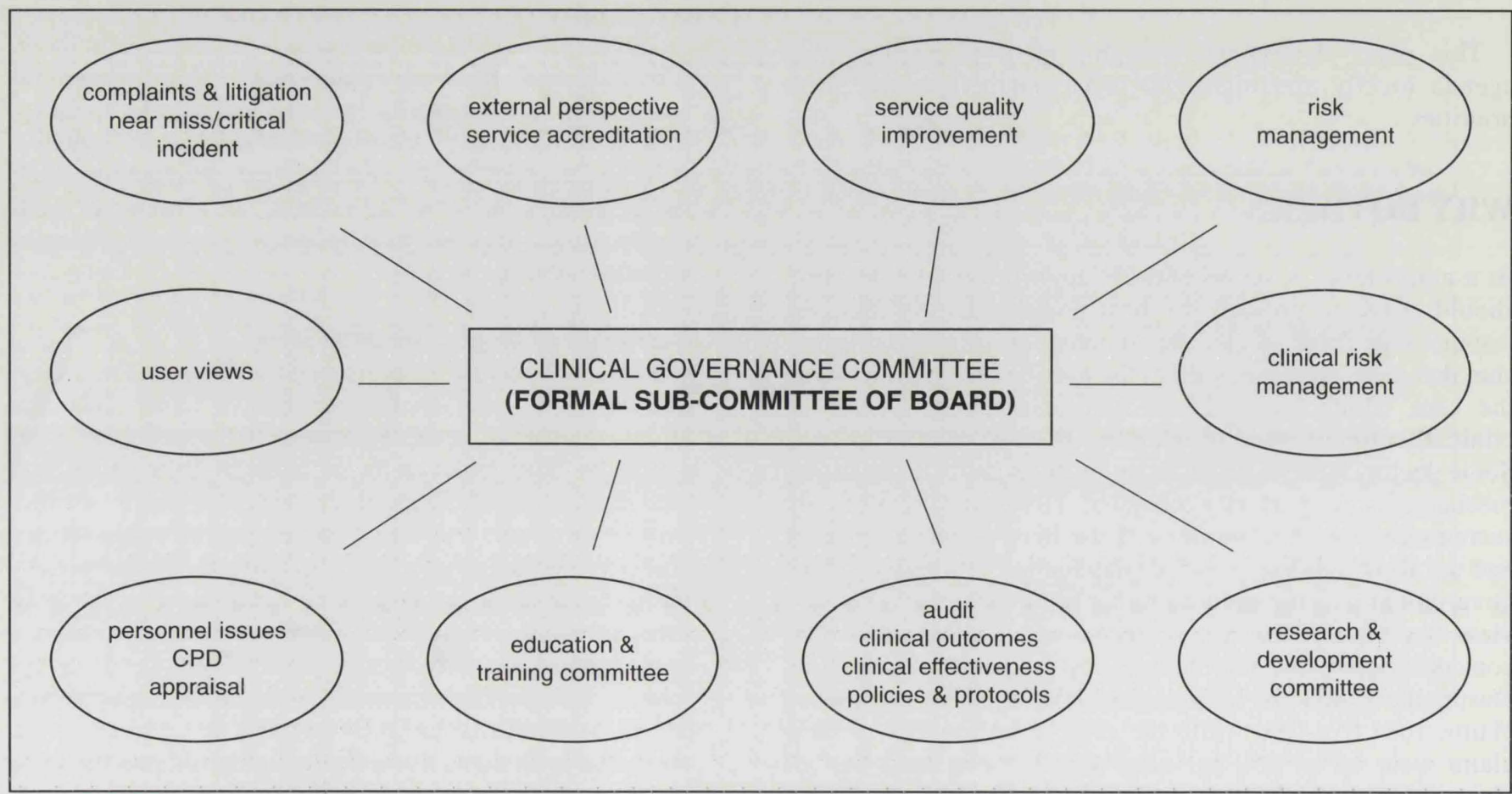

Table 1 Clinical Governance - Morecambe Bay Hospitals Trust approach: groups established 
commitments and extend to discuss a rolling programme of CPD based on the needs of the population served. Thus a three-year rolling programme might include regular time devoted to a doctor's particular special interest and to other relevant fields of study or the learning of new techniques.

Research \& development The ability to practice clinically effective medicine must be based on comprehensive and worthwhile research. Good research alone is not enough since the knowledge must be readily available to clinicians in all parts of the country. There needs to be a systematic approach to the collection and dissemination of evidence, both within our organisation and nationally. In addition to guidelines to be issued from NICE, we already have access to the Cochrane Library and the NHS Centre for Review and Dissemination. The latter body publishes "Effective Health Care Bulletins" and "Effectiveness Matters", publications which are readily available to all trusts. Similarly there is an intention to launch a national electronic library for health which will include summaries of research evidence, including systematic reviews and evidence-based guidelines to support clinical practice.

Poor performance/competency In any system where the majority are proficient there will occasionally be issues surrounding an individual's poor performance or lack of competence. Unfortunately, although such individuals are undoubtedly in the minority, they often have a disproportionate impact. Following the Bristol cardiac surgery problem, the media are increasingly vigilant and are keen to highlight cases of poor practice. To date, mechanisms of recognising and dealing effectively with problems of poor clinical practice have been inadequate. Important new proposals have, however, now been made by the GMC and the Royal Colleges to strengthen procedures so that doctors' rights to remain on the medical register would have to be reaffirmed every three to five years.

Education and training Morecambe Bay Hospitals NHS Trust is determined to foster a culture of learning and has striven to retain training grades and to expand our number of medical students in all disciplines. Having young doctors and students in training encourages a culture of learning and helps keep even the older ones up to date. In the same way, the encouragement of local research and development and a strong culture of multi-disciplinary education enhance the process.

\section{(iv) Individual level}

The majority of doctors working within our trust are genuinely open minded, keen to learn and to improve their services, and clinically competent. Many already participate in clinical audit and work to nationally accepted guidelines. In addition, some directorates already participate in critical incident reporting. We firmly believe the culture of openness and the willingness to learn from near misses or mistakes will prevail. Nevertheless the following may be seen as a very useful checklist which encompasses the responsibilities of an individual in complying with the clinical governance agenda.
a) keep skills and knowledge up to date
b) recognise limits
c) monitor practice (audit)
d) act quickly to protect patients from colleagues whose practice is suspect.

\section{WHEN DOES ALL THIS HAVE TO BE ACHIEVED BY?}

We have described an ambitious vision for the trust. In the short term, these processes must start. In the longer term, and perhaps more importantly, we must move from a culture of blame to one of openness. Nevertheless the government is keen to see a fixed timetable observed and has identified four key steps which must be implemented by April 2000.

- Establish leadership, accountability and working arrangements. The chief executive and the board will be responsible for ensuring that this duty is discharged properly. It is essential that the board demonstrates commitment from the top and makes the process inclusive.

- Carry out baseline assessment of capacity and capability. The trust will have to carry out an assessment of its strengths and weaknesses in relation to quality. The major obstacle is lack of good data and the implementation of improved information technology is vital to the process. Equally it must be recognised that many of the initiatives necessary to fulfill the clinical governance agenda are already in place within the trust (eg audit, risk management, etc).

- Formulating and agreeing the development plan. Having assessed our shortcomings action will need to be taken to address deficiencies.

- Clarifying reporting arrangements. The trust will ensure that it has appropriate mechanisms in place to deliver reports to the board on the progress made in implementing the clinical governance agenda.

\section{WHERE IS THE MONEY COMING FROM?}

A good and as yet unanswered question. Improved information technology is essential to the implementation of clinical governance. It will require both hardware and accurate data collection, which will have resource implications. Similarly, checking that audit is being carried out properly, collating data from critical incident reports and disseminating information may require additional manpower. Appraisal, if it is deemed to be necessary on an annual basis, will also be time-consuming. Finally, if revalidation becomes the norm and individuals are found to need retraining, then the trust may need to meet the costs of both locum cover and reeducation costs. No additional significant recurrent resource for any of the above has yet been identified nationally. There are many things that can, however, be done with existing resources and the lack of targeted funding cannot be a reason for lack of progress locally.

\section{CONCLUSIONS}

This paper has touched on just a few of the issues related to the clinical governance agenda. There are also many other external influences which affect the quality of care provided by the trust. These include accreditation of services by various bodies, the requirements of our insurers, the CNST, and the views of user groups, $\mathrm{CHC}$ and other pressure groups. Table 2 illustrates the varied external influences feeding into the trust.

We firmly believe that, if it is properly funded and appropriately implemented, clinical governance can lead to an improvement in the quality of provision within the NHS. It will guarantee uniformity of standards and raise quality by a 
mechanism of continuous improvement. To succeed it requires a major cultural change, highlighted in the following statement from Jenny Simpson, Chair of the British Association of Medical Managers: "Commitment - first and foremost a change in attitude must be brought about in both the clinical and managerial worlds. Without changing hearts and minds none of the strands of implementation can be put in place."

\section{BIBLIOGRAPHY}

A First Class Service - Quality in the new NHS. NHS White Paper, 1997

Clinical Governance in the new NHS. The British Association of Medical Managers (BAMM), 1998

Clinical Governance: Quality in the new NHS. NHS Executive Circular, 16 March 1996

Good Medical Practice - Duties of a Doctor. General Medical Council

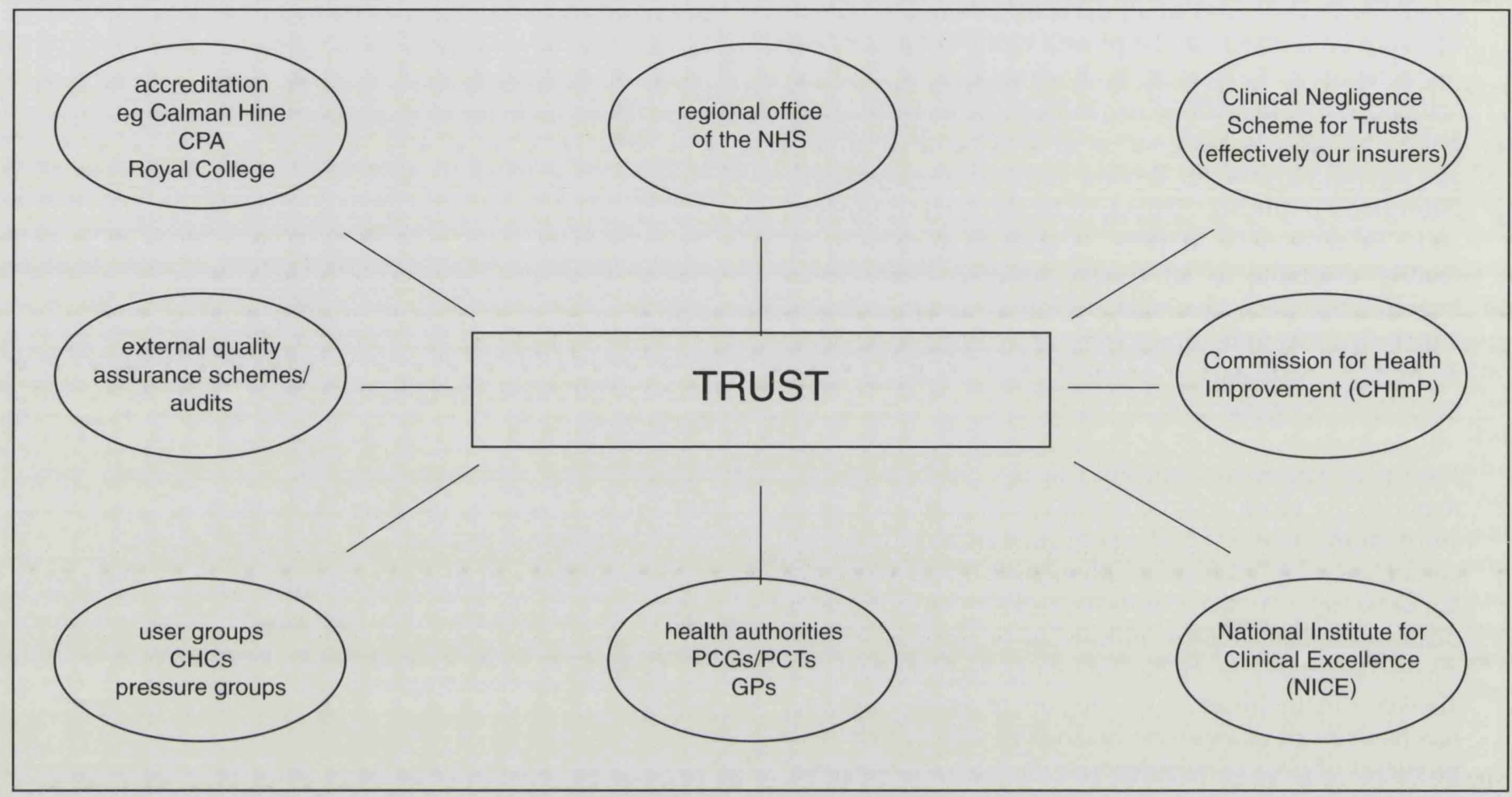

Table 2 Clinical governance - external influences 